\title{
FLORA DE GRÃO-MOGOL, MINAS GERAIS: TURNERACEAE ${ }^{1}$
}

\author{
MARIA MERCEDES ARBO \\ Instituto de Botánica del Nordeste, Casilla de Correo 209, 3400 Corrientes, Argentina.
}

Arbo, M. M. 1995. Turneraceae, Parte I. Piriqueta. Fl. Neotrop. Monogr. 67: 1-156.

Arbo, M. M. 2005. Estudios sistemáticos en Turnera (Turneraceae). III. Series Anomalae y Turnera. Bonplandia (Argentina) 14(3-4): 115-318.

Arbo, M.M. \& P.H. da Silva. 2005. Turneraceae. In M.G.L. Wanderley, M.G.L. et al. (eds.) Flora fanerogâmica do estado de São Paulo. FAPESP, São Paulo, vol. 4, p. 351-359.

Urban, I. 1883. Monographie der familie der Turneraceen. Jahrb. Königl. Bot. Gart. Berlin 2: 1-152.

Urban, I. 1883. Turneraceae. In C.F.P. Martius (ed.) Flora brasiliensis. Frid. Fleischer, Leipzig, vol.13, pars 3: 86-170.

1. Planta con pelos porrecto-estrellados y pelos glandulares setiformes con base bulbosa y cabeza diminuta; pedúnculos florales libres, profilos nulos; flores con una corona membranácea, generalmente fimbriada, anular, inserta en la garganta, sobre la base de la lámina de los pétalos y sobre los sépalos.....

2. Flores amarillas dispuestas en inflorescencias axilares 2-6 floras, las de los nudos inferiores a menudo unifloras; profilos desarrollados; hojas coriáceas, dientes obtusos, glanduloso-impresos, a veces con nectarios diminutos Piriqueta

2'. Flores rosadas o de color salmón, axilares, solitarias, profilos generalmente nulos o muy pequeños; hojas herbáceas, dientes eglandulosos.

3. Flores pequeñas, hasta $12 \mathrm{~mm}$ long., homostilas

P. viscosa subsp. viscosa

3'. Flores grandes, 12-22 mm long., heterostilas. P. duarteana var. duarteana

1'. Planta con pelos simples (raramente con pelos estrellados) y pelos glandulares no setiformes; pedúnculos

florales parcial o totalmente adnatos a los pecíolos, profilos desarrollados; flores sin corona Turnera

4. Flores heterostilas, amarillas o rojas, filamentos estaminales soldados al tubo calicino solamente en la base, por su cara externa; fruto liso.

5. Hierbas con ejes simles a poco ramificados

6. Hierba graminiforme, a veces ramificada, con hojas filiformes o lineares uninervadas T. subnuda

6'. Hierbas no graminiformes, hojas de forma variable, plurinervadas

7. Planta 3-16 cm alt. Hojas opacas, herbáceas, a veces cartáceas, generalmente no pardas en seco, elípticas, ovadas, a veces obovadas T. hilaireana

7'. Planta 10-40 cm alt. Hojas lustrosas, coriáceas, frecuentemente pardas en seco, elípticas, ovadas, a veces lineares

5'. Arbustos o subarbustos ramificados, hojas plurinervadas, discoloras.

8. Pelos estrellados, raramente simples, estípulas $1-3 \mathrm{~mm}$ long.

T. oblongifolia

8'. Pelos simples, estípulas 0,2-0,6 mm long.

9. Flores rojo bermellón con garganta amarilla

T. lamiifolia

9'. Flores amarillas.

10. Hojas con venas secundarias incurvas, dividiéndose y anastomosándose antes del margen con la inmediata superior (venación broquidódroma)

T. coccinea

$10^{\prime}$. Hojas con venas secundarias rectas, terminando en las escotaduras del margen (venación craspedódroma) T. opifera T. aff. uleana

4'. Flores homostilas, amarillas o blancas, filamentos estaminales soldados por sus bordes a la uña de los pétalos hasta la garganta, formando bolsillos nectaríferos; fruto granuloso.

11. Flor amarilla T. orientalis

11 '. Flor blanca, a veces las venas o la garganta violáceas T. candida

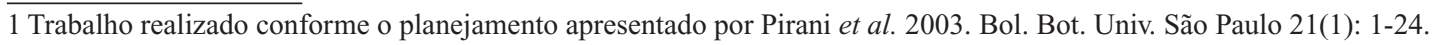




\section{Piriqueta Aubl.}

Hierbas o subarbustos. Hojas generalmente sin nectarios. Flores solitarias, a veces reunidas en cincinos axilares, excepcionalmente en racimo terminal, frecuentemente heterostilas; pedúnculo floral libre; pedicelo desarrollado, articulado; uñas de los pétalos y bases de los filamentos estaminales adnatas al tubo calicino; corona inserta en la garganta. Cápsula lisa o verrucosa. Semilla reticulada; arilo inserto alrededor del hilo, carnoso en vivo, membranáceo en seco, unilateral o envolvente.

1.1. Piriqueta duarteana (A. St.-Hil., A. Juss. \& Cambess.) Urb., Jahrb. Königl. Bot. Gart. Berlin 2: 66. 1883.

Hierba o subarbusto, 20-75 cm alt., ramas nuevas con indumento igual o más corto que el diámetro de los tallos. Hojas viscosas, pecíolo 2-16 mm long., lámina ovada o angustiovada, a veces suborbicular, 6-55 mm long., 5-32 $\mathrm{mm}$ ancho. Pedúnculo floral 2-10(-15) mm long.; profilos nulos, a veces desarrollados hasta $2 \mathrm{~mm}$ long.; pedicelo 1-10 mm long.; cáliz 7-15 mm long., corola rosada o color salmón. Cápsula tuberculada con pelos glandulares de base clara u oscura. Semilla 2-2,6 mm long., reticulada, epidermis generalmente pilosiúscula. (Fig. 1 A-B)

Campos et al. CFCR 13520 (SPF); Cavalcanti et al. CFCR 9581 (CTES, SPF); Esteves \& Kameyama 2515 (SP); Freire-Fierro et al. CFCR 12352 (CTES, SPF); Hatschbach 41250 (CTES, NY; Irwin et al. 23370 (NY); Semir et al. CFCR 9582 (CTES, SPF); Silva et al. CFCR 12584 (CTES, SPF); Silva et al. CFCR 12624 (SPF).

Especie representada en Grão-Mogol por P. duarteana var. duarteana, variedad conocida de Pará, Tocantins, Maranhão, Piauí, Ceará, Pernambuco, Rio Grande do Norte, Goiás, Bahia y Minas Gerais, en cerrado, campo rupestre, carrascal y bosque abierto. En Grão-Mogol se la coleccionó desde noviembre hasta abril entre los 700 y 1650 m de elevación.

1.2. Piriqueta sidifolia (A. St.-Hil., A. Juss. \& Cambess.) Urb., Jahrb. Königl. Bot. Gart. Berlin 2: 61. 1883.

Subarbusto 0,2-1 m alt., hojas coriáceas, discoloras, abolladas, pecíolo 0,5-0,7 mm long., lámina 3-10 cm long., 1,2-6 cm ancho, ovada o elíptica. Inflorescencias cimosas, 2-3(-6)-flo- ras, las inferiores a menudo unifloras; profilos desiguales, 1-5 mm long., opuestos o alternos. Flores heterostilas, cáliz 7-15 mm long., corola amarilla, más larga que el cáliz. Cápsula con dorso tuberculado o verrucoso. Semilla obovoide, recta o ligeramente curvada, epidermis pilosiúscula. (Fig. 1 C-D)

Hatschbach et al. 54232 (CTES).

En Grão-Mogol se encuentra solamente P. sidifolia var. sidifolia, conocida de cerrados y campos de Bahia, Goiás, Tocantins y Minas Gerais; florece de septiembre a junio. En Grão-Mogol ocurre en campo rupestre de suelo rocoso.

\subsection{Piriqueta viscosa Griseb., Cat. pl. Cub. 114. 1862.}

Hierba poco ramificada, $8-55 \mathrm{~cm}$ alt. Hojas herbáceas, viscosas, pecíolo 1-8 mm long., lámina elíptica o angustiovada 13-54 mm long., 3.5-22 mm ancho, haz con pelos glandulares y porrecto-estrellados pauci-radiados. Pedúnculo floral 15$45 \mathrm{~mm}$; pedicelo 3-6 mm long.; cáliz 3.5-7 mm long., corola rosada, 1-5 mm más larga que el cáliz. Cápsula verrucosa o granulosa. Semillas delgadas, 1,6-2,2 x 1,6-0,8 mm, retículo de aréolas pequeñas, epidermis glabra. (Fig. 1 E-F)

\section{Silva et al. CFCR $12576(\mathrm{SPF})$.}

En Grão-Mogol se encuentra solamente $P$. viscosa subsp. viscosa, la cual vive en Antillas y Mesoamérica en bosques de pinos y sabanas, en América del Sur en sabanas y lugares abiertos frecuentemente de suelo arenoso desde Venezuela y Guayanas hasta Bolivia y Mato Grosso do Sul. En Grão-Mogol colectada en el valle del río Itacambiruçu a $1650 \mathrm{~m}$.

\section{Turnera Plum. ex L.}

Hierbas o arbustos. Hojas frecuentemente con nectarios. Flores solitarias, a veces reunidas en cimas o racimos capituliformes, a menudo heterostilas; pedúnculo floral frecuentemente soldado al pecíolo; profilos desarrollados; pedicelo frecuentemente ausente; pétalos con la uña soldada al tubo calicino. Cápsulas lisas o verrucosas. Semillas frecuentemente reticuladas, a veces estriadas o crestadas; arilo inserto alrededor del hilo, carnoso en vivo, membranáceo en seco, unilateral o envolvente.

2.1. Turnera candida Arbo, Bonplandia 7: 68, fig. 2. 1993; Arbo, Bonplandia 14: 199-200. 2005.

Sufrútice 50-60 cm alt. Hojas aserradas, pecíolo 3-7 $\mathrm{mm}$ long., con un par de nectarios en el ápice, lámina elíptica $u$ ovada, 2-7 cm long., 6-26 mm ancho, haz con pelos simples muy cortos, pelos glandulares y pelos simples largos oblicuos. Flores epifilas, pedicelo nulo, profilos 5-12 mm long.; cáliz 11-19 mm long., corola 4-7 mm más larga que el cáliz; con bolsillos nectaríferos entre cada filamento estaminal y el cáliz. Cápsula verrucosa. Semilla recta, obovoide, 2,1-3 mm long., 0,7-1,2 mm diám., reticulada, aréolas más o menos cuadrangulares. (Fig. 1G)

Hatschbach et al. 55062 (CTES, MBM); Silva et al. CFCR 13522 (SPF). 


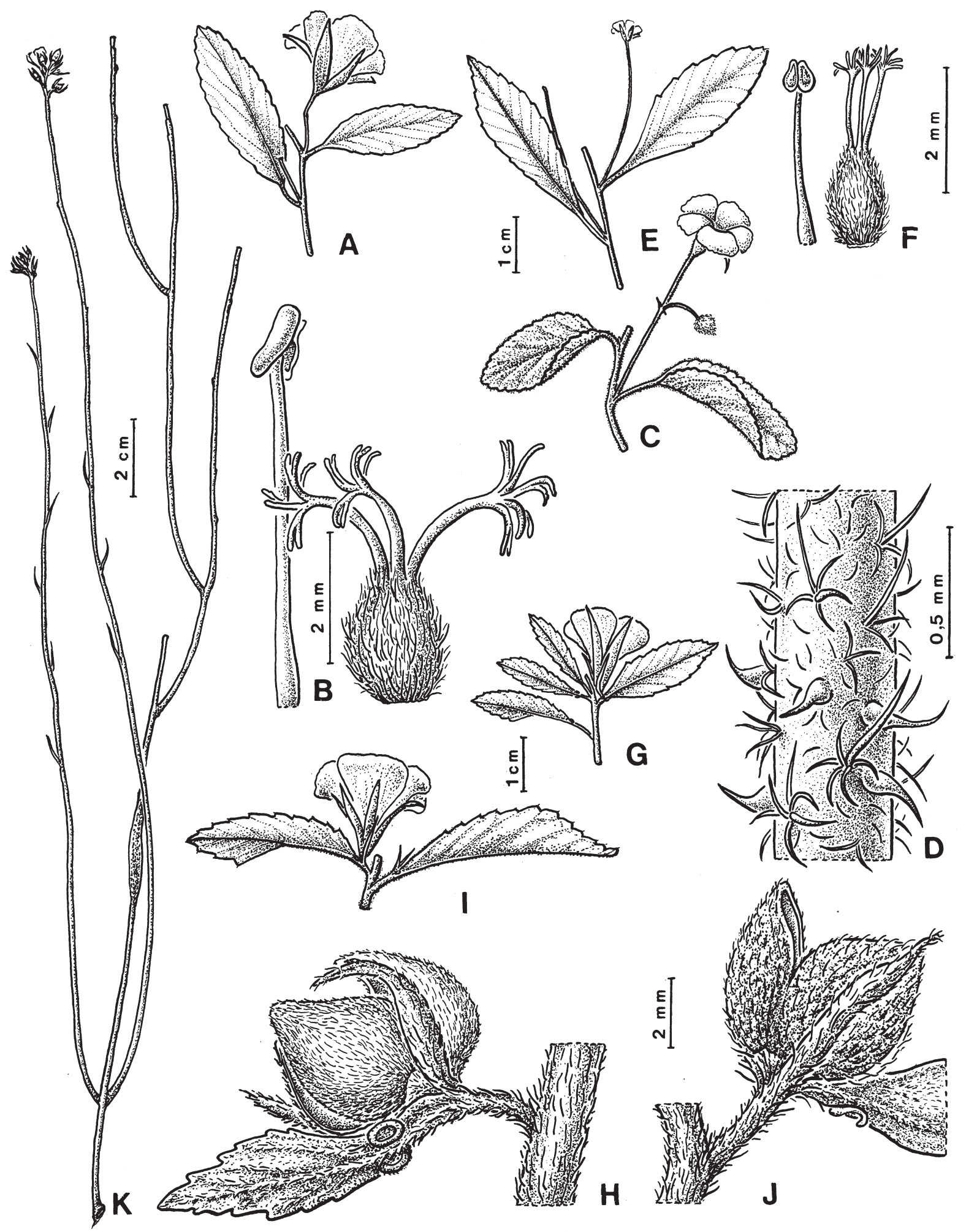

Fig. 1. TURNERACEAE. A-B. Piriqueta duarteana var. duarteana. A, rama con flor axilar solitaria. B, flor brevistila, estambre y gineceo. C-D. P. sidifolia var. sidifolia. C, rama con inflorescencia axilar biflora. D, porción de tallo con pelos porrecto-estrellados, simples y glandulares. E-F. P. viscosa subsp. viscosa. E, rama con flor axilar solitaria. F, flor homostila, estambre y gineceo. G, Turnera candida, rama con flor epifila. H, T. opifera, nudo y hoja con fruto y profilos sobre la haz y mostrando los nectarios en el envés. I-J. Turnera orientalis. I, porción de rama con flor epifila. J, nudo y porción basal de una hoja con fruto y profilo sobre la haz y mostrando un nectario en el borde. K, T. subnuda, planta. 
Propia de Bahía, Piauí y Minas Gerais, en campo rupestre, cerrado y caatinga, suelos arenosos. Florece y fructifica desde octubre hasta mayo. En Grão-Mogol, coleccionada en área de cerrado perturbado.

\subsection{Turnera coccinea Arbo, sp. nov.}

Turnera coccinea $s p$. nov. serie Leiocarpae, a ceteris speciebus serie combinatione frutex globosus usque $1,5 \mathrm{~m}$ altis, foliis 1-2,6-plo longioribus quam latioribus, floribus verisimiliter dimorphis, coccineis basi intra lutea differt.

TYPUS. Brasil. Minas Gerais: extremo N de Serra da Bocaina, próximo a las nacientes del río Ventania, aprox. 16²'S'S 4253'W, 920 m, 20.XI.1992, R. Mello-Silva, M.M. Arbo \& J. Vicente Nunes 760 (holotypus SPF; isotypus CTES).

Arbusto globoso 0,7-1,5 m, tallos erectos 2,5-4 mm diám., densamente estrigosos, a veces tomentosos hacia el ápice, pelos amarillos, gruesos, curvado-antrorsos, mezclados con pelos cortos crespos. Hojas discoloras. Estípulas 2-3 a cada lado, 0,2-0,5 mm long., subuladas. Pecíolo 2-5 mm long., indumento como el del tallo. Lámina elíptica, ovada, obovada, suborbicular, orbicular, 9-27 x 6-20 mm, relación largo:ancho = 1-2,6:1; base redondeada o cuneada, ápice agudo u obtuso, margen revoluto, aserrado-crenado excepto en la porción basal; haz pardo-obscura en seco, estrigosa, superficie irregular debido a la forma cónica de la base de los pelos, venas deprimidas; envés tomentoso-velutino, dorado, con pelos glandulares capitados amarillos subsésiles entremezclados; 4-5 pares de venas rectas o ligeramente incurvas, ángulo de divergencia ca. $45^{\circ}$, algunas bifurcándose más o menos a la mitad de la lámina, terminando en las escotaduras del borde o dividiéndose antes y con una de las ramas dirigida a la escotadura; venas terciarias recurvas, visibles en el haz. Nectarios 1+1, circulares, 0,6-0,7 mm diám., borde pilosiúsculo, parte central con una membrana, situados en el envés de la lámina, sobre el margen. Flores probablemente dimorfas (sólo se observó la forma longistila), epifilas, reunidas en los ápices de las ramas; hojas floríferas con pecíolo y envés rojo, de tamaño bruscamente decreciente, hasta 6-4 mm long., con un par de nectarios grandes, pardos o negruzcos, 0,6-1 mm diám. Pedúnculo 1,5-3 mm long., totalmente adnato al pecíolo. Profilos lineares o subulados, rojos, 3-4 x 0,5 mm, insertos en la base del receptáculo. Cáliz vinoso, 8-11 mm long., con pelos simples, cortos y erectos, pelos curvado-antrorsos y pelos glandulares capitados; tubo 4-5 mm long., interiormente velloso; lóbulos 3-5 nervados, con pelos de igual longitud que los del tubo, los externos pilosos hasta el borde, los internos con borde membranáceo, ancho y glabro, nervio medio prolongado en mucrón 0,5 mm long. Corola rotácea, 11-17 mm long., 3-6 mm más larga que el cáliz, rojo bermellón con garganta amarilla, pétalos con uña de $3 \mathrm{~mm}$ long., lámina obovada, 8-9 x 4 mm. Filamentos estaminales glabros, subulados, soldados $1 \mathrm{~mm}$ al tubo en la porción basal, 5-6 mm long. en flores longistilas; anteras ovado-elípticas, $1,5 \times 1 \mathrm{~mm}$, base emarginada, ápice obtuso o apiculado. Ovario cónico, densamente piloso, 1,5-2 x 1,3 mm, placentas 4-6-ovuladas; estilos cilíndricos, 4-6 mm long. en flores longistilas, porción basal glabra, porción distal partida en 2-4 ramas pilosas; estigmas paucirramosos, $2 \mathrm{~mm}$ long., ramas 2-3 veces divididas. Fruto 6-8 mm diám., subesférico o cónico, valvas castañas por fuera, con pelos largos, adpresos, laxos, entremezclados con pelos cortos, crespos, y pelos glandulares capitados subsésiles, por dentro glabras, nítidas, jaspeadas. Semilla 2,6-3,7 x 1,2-1,5 mm, obovoide, recta o ligeramente curvada, base atenuada, parda, reticulada, nudos no prominentes, aréolas muy pequeñas, epidermis con cera epicuticular, exóstoma redondeado, 0,3 mm long., rafe linear saliente, cálaza ligeramente prominente, pigmentada; arilo más corto que la semilla, unilateral, blanquecino en seco en la porción basal.

Paratypi. BRASIL. Minas Gerais: Caveira, BR-251, 41 $\mathrm{km}$ da entrada para Grao Mogol 27.III.1993, G.L. Esteves \& C. Kameyama 2510 (SP); Grão-Mogol, extremidade N da Serra da Bocaina, na rodovia BR-251, nascente do Rio Ventania, 12.VI.1991, R. Mello-Silva et al. 452 (CTES, SPF).

Especie aparentemente endémica de la región de GrãoMogol, donde fue recolectada en marzo, junio y noviembre. Vegetativamente es muy afín a los ejemplares de Turnera opifera con hojas pequeñas; el material seco, sin flores, es difícil de diferenciar. T. coccinea y T.opifera son las especies de mayor porte en la Serie Leiocarpae, a la cual pertenecen.

Las flores de color rojo bermellón son muy raras en el género, seguramente están asociadas a polinizadores diferentes. La única otra entidad de la serie Leiocarpae con flores de ese color es T. longiflora Cambess., arbusto virgado hasta de $1 \mathrm{~m}$ alt., con flores grandes (hasta $3 \mathrm{~cm}$ long.), hojas hasta $10 \mathrm{~cm}$ long., relación largo:ancho = 1,9-3,6:1, que vive en el cerrado y en campos rupestres, en el Distrito Federal, Goiás y zona limítrofe de Minas Gerais.

\subsection{Turnera hilaireana Urban, Jahrb. Königl. Bot. Gart. Ber-} lin 2: 108-110. 1883.

Hierba erguida 3-17 cm alt., tallos con pelos simples cortos antrorsos y pelos simples largos hasta $2 \mathrm{~mm}$ long. Hojas herbáceas, generalmente opacas; pecíolo 0-1,5 mm long., lámina ovada, elíptica u obovada, 7-28 x 4-15 mm, con pelos simples, a veces sólo las venas pilosas; nectarios 2, en la base, sobre el envés. Flores solitarias, epifilas, heterostilas; profilos lineares o subulados, 1-3,5 mm long., cáliz 6-10 mm long., corola amarilla, más larga que el cáliz. Cápsula lisa, 3-4 mm diám. Semilla obovoide, 1,6-2,3 x 0,8-1,2 mm, reticulada; arilo unilateral.

Markgraf et al. 3223 (F).

Nativa en el Distrito Federal, Goiás, Minas Gerais y São Paulo, propia de campos y cerrados. En Grão-Mogol colectada a $1000 \mathrm{~m}$ de altitud. 


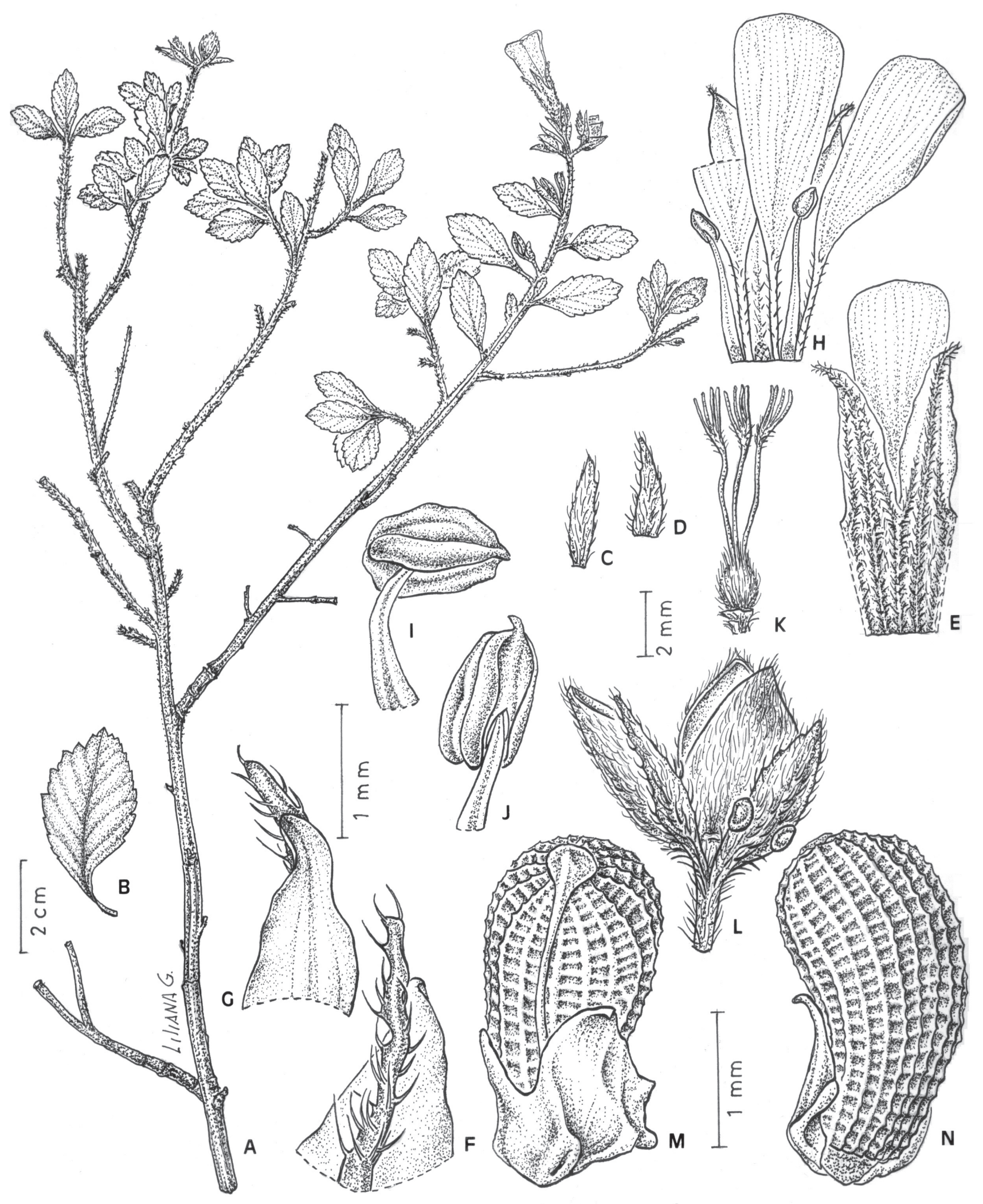

Fig. 2. TURNERACEAE. A-N. Turnera coccinea. A, rama con flores axilares solitarias. B, hoja. C-D, profilos. E, porción del cáliz, cara externa, y un pétalo. F-G, ápice de un lóbulo del cáliz, cara externa e interna. H, flor heterostila, porción del cáliz, cara interna, con 3 pétalos y 2 estambres adnatos; de ha cuadriculado la cicatriz dejada al desprender un estambre. I-J, antera, vista lateral y dorsal. K, gineceo. L, fruto epifilo y profilo, hoja tectriz con nectarios. $\mathrm{M}-\mathrm{N}$, semilla y arilo, vista rafeal y cara opuesta. 
2.4. Turnera lamiifolia Cambess. in A. St.-Hil., A. Juss. \& Cambess., Fl. Bras. merid. 2: 155 (213). 1830.

Subarbusto $15-30 \mathrm{~cm}$ alt., indumento blanquecino o amarillento, pelos simples largos y pelos estrellados densos. Hojas abolladas, discoloras, pecíolo 3-12 mm long., lámina ovada u obovada, 5-12 cm long., 3-6 cm ancho, con un par de nectarios oscuros, haz oscura, hirsuta a tomentosa, envés lanoso-tomentoso. Flores heterostilas, agrupadas hacia los ápices, hojas floríferas de tamaño bruscamente decreciente, con nectarios de mayor tamaño; profilos lineares o subulados, 5-10 mm long., cáliz 10-12 mm long., corola amarilla, más larga que el cáliz. Cápsula lisa, 5-7 mm diám. Semilla obovoide, 2,8-3 mm long., 1,2-1,4 mm diám., tenuemente reticulada.

Irwin et al. $23418(\mathrm{MO}, \mathrm{P})$.

Propia de Goiás y Minas Gerais, característica del cerrado. En Grão-Mogol, coleccionada a $950 \mathrm{~m}$ de altitud.

2.5. Turnera oblongifolia Cambess. in A. St.-Hil., A. Juss. \& Cambess., Fl. Bras. merid. 2: 155 (215). 1830.

Hierba perenne erecta, 10-40 cm alt., tallos cilíndricos. Hojas coriáceas, lustrosas, erguidas, con pelos simples, con un par de nectarios en la base, sobre el envés, pecíolo 0-1,5 mm long., lámina elíptica, ovada, a veces lanceolada, 1,3-5 cm long., 0,3-1,1 cm de ancho, venación pinada. Flores heterostilas, epifilas, profilos lineares 1,2-7 mm long., pedicelo nulo, cáliz 5-7 mm, corola amarilla, más larga que el cáliz. Cápsula lisa, 3-5 mm diám. Semilla obovoide, 1,5-1,6 mm long., reticulada, aréolas rectangulares, arilo unilateral.

Harley et al. CFCR $6420(\mathrm{~K})$.

Propia de las regiones montañosas de Bahía, Goiás, Minas Gerais, São Paulo y Paraná, donde crece en campos y cerrados. En Grão-Mogol, fue colectada en la Serra do Calixto, a $1050 \mathrm{~m}$ de altitud.

\subsection{Turnera opifera Mart., Reise Bras. 2: 552. 1828.}

Subarbusto o arbusto 0,25-1,50 m alt., indumento amarillento. Hojas discoloras, pecíolo 2-12 mm long., lámina elíptica, obovado-oblonga o lanceolada, 2,5-11 cm long., 1-4 cm ancho, con 1-3 pares de nectarios, haz oscura, lustrosa, pilosa; envés con indumento denso, pelos simples y pelos glandulares microcapitados amarillos. Flores heterostilas, epifilas, agrupadas hacia los ápices; hojas floríferas de tamaño bruscamente decreciente, hasta 6-2 mm long., con nectarios grandes; profilos lineares o subulados, 2,5-5,5 mm long., cáliz verde-amarillento, 5,5-13 mm long., corola amarilla, 3-7 mm más larga que el cáliz. Cápsula lisa, 5-8 mm diám. Semilla obovoide, 2,3-3 mm long., reticulada, arilo lateral (Fig. 1H)
Cordeiro et al. CFCR 818 (CTES, MBM, SPF); Harley et al. CFCR 6491 (CTES, K, SPF); Harley et al. 25096 (CTES, K, SPF); Hatschbach 41404 (CTES, MBM, SPF, US); Hatschbach et al. 54194 (C, CTES, DAV, MBM, MO); Hatschbach et al. 67966 (C, CTES, MBM); Hensold et al. 3509 (CTES, SPF); Irwin et al. 23418 (CTES, UB); Irwin et al. 23596 (NY, P, UB); Markgraf et al. 3427 (F, R, RB); Mello-Silva et al. s.n. CFCR 9695 (UEC) y 11396 (CTES, SPF); Mello-Silva et al. 749 (CTES, SPF); Pirani et al. CFCR 896 (CTES, F, SPF) y 9018 (CTES, SPF); Semir et al. CFCR 9703 (CTES, SPF); Silva et al. CFCR 12392 (CTES, SPF; Simão-Bianchini et al. CFCR 13145 (CTES, SPF).

Especie con variabilidad foliar muy marcada, algunos ejemplares presentan hojas de lámina muy amplia, mientras otros poseen sólo hojas pequeñas; los rebrotes presentan hojas herbáceas y angostas. Los órganos vegetativos de los ejemplares de hojas pequeñas son muy similares a los de $T$. coccinea.

Entidad propia de cerrados y campos rupestres, a veces también se halla en borde de bosque, sobre suelos rocosos o arenosos, a veces arcillosos. Crece bien en terrenos modificados. Vive en el norte de Minas Gerais y en la zona limítrofe de Bahia, entre los 750 y 1300 m de elevación. En Grão-Mogol florece desde octubre hasta junio.

\subsection{Turnera orientalis (Urb.) Arbo, Candollea 40: 177. 1985.}

Sufrútice erecto 0,2-1,5 m alt., ramificado. Hojas herbáceas, pecíolo 2-18 mm long., con un par de nectarios en el ápice, lámina ovada, elíptica o lanceolada, 1,5-9 cm long., 6$42 \mathrm{~mm}$ ancho, margen aserrado-crenado. Flores homostilas, epifilas, hojas floríferas bien desarrolladas, profilos subulados, 4,5-13 mm long., cáliz 11-15 mm long., corola amarilla, más larga que el cáliz, con bolsillos nectaríferos entre cada filamento estaminal y el cáliz. Cápsula granulosa, 4,5-10 mm diám. Semilla claviforme, 2,3-2,7 mm long., reticulada, aréolas subcuadrangulares. (Fig. 1 I-J)

\section{Silva et al. CFCR 13305 (CTES, SPF).}

Especie de amplia distribución, vive en Bolivia, en la región centro-oriental de Brasil desde Tocantins y Bahia hasta Paraná, en Paraguay y en el noreste argentino. Florece casi todo el año. En Grão-Mogol se la coleccionó en Noviembre.

2.8. Turnera subnuda Urb., Bot. Jahrb. Syst. 25, Beibl. 40: 8. 1898.

Hierba perenne erecta, 30-75 cm alt., tallos cilíndricos. Hojas erguidas, las floríferas con un par de nectarios, pecíolo 0-1,5 mm long., lámina filiforme, linear o angustiovada, 3-15 mm long., 0,4-1,5 mm ancho, uninervada, haz glabra. Flores heterostilas, epifilas, profilos subulados 1,2-2,5 mm long., cáliz 5-7 mm, corola amarilla, más larga que el cáliz. Cápsula lisa, 3-5 mm diám. Semilla obovoide, 1,5-1,6 mm long., reticulada, aréolas rectangulares (Fig. $1 \mathrm{~K}$ )

Hatschbach et al. 54229 (C, CTES, MU). 
Especie rara, de la que se han coleccionado unos pocos ejemplares en Goiás y Minas Gerais. Crece sobre rocas serpentinas. Florece y fructifica desde octubre a junio.

2.9. Turnera aff. uleana Urb., Fedde Repert. Spec. Nov. Regni Veg. 4: 132 (1907).

Subarbusto erecto o tortuoso, 20-80 cm alt. Hojas patentes o recurvadas, extendidas o plegadas longitudinalmente en seco, discoloras, lámina de 2,2-4,5 x 0,6-1,4 cm, margen crenado a marcadamente dentado, haz lustrosa, verde oscura, con pelos laxos a velutina, envés verde grisáceo a plateado, desde glabro excepto en las venas hasta seríceo-lanoso; venas secundarias rectas, terminando en las escotaduras del margen. Flores heterostilas, epifilas. Profilos 3,5-6 mm long. Cáliz amarillo, 7-10 mm long., corola ca. $4 \mathrm{~mm}$ más larga que el cáliz, pétalos amarillo vivo con venas anaranjadas en la cara externa o con mácula vinosa en la base. Cápsula lisa. Semilla reticulada, 2,7 x 1,2 mm; arilo unilateral.

Hatschbach 41241 (CTES, MBM); Hatschbach et al. 56164 (MBM, MU); Pirani et al. CFCR 987 (CTES).

Taxón nativo de Bahía y Minas Gerais, vive en carrasco, cerrado, campos gerais y campo rupestre con suelo arenoso o pedregoso, también se adapta a terrenos alterados como bordes de caminos. En Grão-Mogol ha sido coleccionada a $1000 \mathrm{~m}$ de altitud. Puede tratarse de una especie nueva, pero aún está en estudio. 